\title{
Immunohistochemistry as an accurate tool for the assessment of BRAF V600E and TP53 mutations in primary and metastatic melanoma
}

\author{
STEFAN RUSU ${ }^{1}$, CAMILLE VEROCQ ${ }^{1}$, ANNE LAURE TREPANT ${ }^{1,2}$, CALLIOPE MARIS $^{1,2}$, NANCY DE NÈVE ${ }^{1}$, \\ ORIANE BLANCHARD ${ }^{1}$, CLAUDE VAN CAMPENHOUT ${ }^{1}$, SARAH DE CLERCQ $^{1}$, SANDRINE RORIVE ${ }^{1,2}$, \\ OVIDIU SIMION COTOI ${ }^{3}$, CHRISTINE DECAESTECKER $^{4,5}$, ISABELLE SALMON $^{1,2,4}$ and NICKY D'HAENE ${ }^{1,2}$ \\ ${ }^{1}$ Department of Pathology, Erasme University Hospital, Université Libre de Bruxelles, B-1070 Brussels; \\ ${ }^{2}$ Centre Universitaire Inter Regional d'Expertise en Anatomie Pathologique Hospitalière (CurePath), \\ B-6040 Charleroi (Jumet), Belgium; ${ }^{3}$ Department of Pathology, Clinical County Hospital of Targu Mures, \\ University of Medicine, Pharmacy, Science and Technology 'George Emil Palade' of Targu Mures, \\ Targu Mures, RO-540139 Mures County, Romania; ${ }^{4}$ DIAPath-Center for Microscopy and Molecular Imaging, \\ Université Libre de Bruxelles, B-6041 Gosselies; ${ }^{5}$ Laboratory of Image Synthesis and Analysis, \\ Ecole Polytechnique de Bruxelles, Université Libre de Bruxelles, B-1050 Brussels, Belgium
}

Received September 25, 2020; Accepted July 15, 2021

DOI: $10.3892 / \mathrm{mco} .2021 .2432$

\begin{abstract}
Metastatic melanoma is a fatal disease with poor prognosis. Ever since targeted therapy against oncogenic $B R A F$ was approved, molecular profiling has become an integral part of the management of such patients. While molecular testing is not available in all pathology laboratories, immunohistochemistry (IHC) is a reliable screening option. The major objective of the present study was to evaluate whether IHC detection of BRAF and the tumor (suppressor) protein 53 gene (TP53) are reliable surrogates for mutation detection. Formalin-fixed paraffin-embedded samples of melanomas for which molecular data were previously obtained by targeted next-generation sequencing (NGS) between January 2014 and February 2019 were immunostained with BRAF V600E and p53 antibodies. A blinded evaluation of the IHC slides was performed by two pathologists in order to evaluate inter-observer concordance (discordant cases were reviewed by a third observer). The associations between the results of IHC and molecular profiling were evaluated. The study included a series of 37 cases of which 15 harbored a BRAF mutation and five a TP53 mutation. IHC had an overall diagnostic accuracy of $93.9 \%$ for $B R A F$ V600E and $68.8 \%$ for TP53 compared to NGS. A statistically significant association between the two diagnostic methods
\end{abstract}

Correspondence to: Dr Nicky D'Haene, Department of Pathology, Erasme University Hospital, Université Libre de Bruxelles, 808 Route de Lennik, B-1070 Brussels, Belgium

E-mail: nicky.d.haene@erasme.ulb.ac.be

Key words: melanoma, BRAF, TP53, immunohistochemistry, next-generation sequencing was obtained for BRAF V600E $(\mathrm{P}=0.0004)$ but not for $\mathrm{p} 53$ $(\mathrm{P}=0.3098) \mathrm{IHC}$. The $\kappa$ coefficient for IHC assessment of $\mathrm{p} 53$ was 0.55 and that for BRAF V600E was 0.72. In conclusion, the present results evidenced that IHC staining is a reliable surrogate for NGS in identifying the BRAF V600E mutation, which may become an efficient screening tool. Aberrant expression of p53 on IHC is at times associated with TP53 mutations but it was not possible to establish a direct link.

\section{Introduction}

Melanoma represents a minority of skin cancers but it is associated with an increased risk of death compared with other skin malignancies (1-4), being responsible for $\sim 90 \%$ of mortalities reported in cutaneous tumors (5). The worldwide incidence has risen rapidly over the last five decades (1-4). Among all newly diagnosed cancers, melanoma was the 17th most common type according to World Cancer Research Fund International in 2019 (6). Melanoma is a heterogeneous tumor with alterations in specific genes involved in pathways controlling cell proliferation, differentiation and survival (7). The major pathways involved are the cyclin-dependent kinase inhibitor 2A (CDKN2A)-dependent pathway and MAPK- and PI3K-dependent pathways $(8,9)$.

As $\sim 50 \%$ of advanced melanomas [unresectable Stage III or metastatic Stage IV, according to the American Joint Committee on Cancer Staging Manual, 8th edition (3)] harbor a mutation in the $B R A F$ gene and since the Federal Drug Administration and the European Institutions approved targeted therapy with BRAF and MAPK kinase inhibitors, molecular testing for codon $V 600$ of the $B R A F$ oncogene became an integral part of the management of such cases. Several approaches are available, from single gene testing for the BRAF V600 mutation, such as PCR or immunohistochemistry (IHC) 
using anti-BRAF (mutated V600E) antibody (AB) [VE1 clone (BRAF VE1)], to whole-genome sequencing. The targeted next-generation sequencing (NGS) technique allows the detection of abnormalities across multiples genes. The identification of multiple molecular aberrations provides several treatment targets. Despite the valuable provided data, NGS remains expensive and not widely distributed and requires sophisticated bioinformatics systems, fast data processing and large data storage capabilities. On the other hand, IHC has a shorter turnaround time (TAT), is cheaper and widely available, but it detects only specific antigens and false-positive and -negative results may be obtained. IHC using BRAF VE1 has emerged as a powerful tool in assessing the BRAF V600E mutation status; validation studies performed to date reported high specificity (95.4-100\%) and sensitivity (94.4-100\%) (10-14).

The tumor (suppressor) protein 53 gene (TP53), 'the guardian of the genome', is inactivated in $~ 90 \%$ of melanomas and only $10-20 \%$ of cases are carrying disabling point mutations (15), but its role remains controversial in melanoma and melanoma progression. IHC is used routinely as a surrogate for TP53 mutation analysis, particularly in ovarian cancer (16), Barrett's esophagus (17) and other cancer types (18-22). Its application in melanoma requires further study (23-29).

In light of the importance of identifying the molecular profile for therapeutic purposes, the main objective of the present study was to evaluate whether BRAF VE1 and p53 immunoexpression are reliable surrogates for mutation detection. For this purpose, BRAF and TP53 status were analyzed by IHC in a series of melanoma cases for which molecular data were previously obtained by NGS. Furthermore, the inter-observer concordance for the IHC assessment was assessed and a cost-effectiveness analysis for the BRAF VE1 $\mathrm{AB}$ was performed.

\section{Materials and methods}

Samples. Formalin-fixed paraffin-embedded (FFPE) samples of 37 melanomas from 37 patients were retrospectively analyzed, including $8(21.6 \%)$ primary tumors and $29(78.4 \%)$ metastatic tumors. The samples were provided by the Pathology Department of Erasme University Hospital (Brussels, Belgium). All tumor sample sites are summarized in Table SI.

The selection criteria were as follows: Patients with melanoma for whom molecular testing was required and performed between January 2014 and February 2019. The sample types were either biopsies $(n=6)$, cell blocks $(n=3)$ or surgical resections $(n=28)$. The samples were retrieved for the NGS results and subsequently, the FFPE blocks were checked to assess if there is sufficient residual tissue to perform IHC. Thus, the exclusion criterion was an insufficient amount of residual tumor tissue as determined by the pathologist and the cases were further excluded from the statistical analyses.

\section{$N G S$}

DNA extraction. DNA extraction from FFPE samples was performed as previously described $(30,31)$, using the QIAamp FFPE tissue kit (Qiagen $\mathrm{GmbH}$ ), according to the manufacturer's protocol. The obtained DNA was quantified using the
Qubit ${ }^{\circledR}$ fluorometer in combination with the Qubit ${ }^{\circledR}$ dsDNA HS assay kit (Thermo Fisher Scientific, Inc.).

Library preparation, cluster amplification and sequencing. NGS was performed as previously described (30,31). DNA (10 ng) was amplified using the Cancer Panel (Ampliseq ${ }^{\mathrm{TM}}$; Thermo Fisher Scientific, Inc.). An amplicon library was generated for sequencing 2,850 mutations in 50 genes, including the following: $A B L 1, A K T 1, A L K, A P C, A T M, B R A F, C D H 1$, CDKN2A, CSF1R, CTNNB1, EGFR, ERBB2, ERBB4, EZH2, FBXW7, FGFR1, FGFR2, FGFR3, FLT3, GNA11, GNAQ, GNAS, HNF1A, HRAS, IDH1, IDH2, JAK2, JAK3, KDR, KIT, KRAS, MET,MLH1,MPL,NOTCH1,NPM1,NRAS, PDGFRA, PIK3CA, PTEN, PTPN11, RB1, RET, SMAD4, SMARCB1, SMO, SRC, STK11, TP53 and VHL. Library construction was performed using the Ion AmpliSeq ${ }^{\mathrm{TM}}$ Library kit 2.0 and Ion Xpress $^{\mathrm{TM}}$ barcode adapters kit (Thermo Fisher Scientific, Inc.), while the quantification was performed with the Qubit ${ }^{\circledR}$ fluorometer and the Qubit ${ }^{\circledR}$ dsDNA HS assay kit (Thermo Fisher Scientific, Inc.) according to the manufacturer's protocol. The library was then quantified using the Qubit ${ }^{\circledR}$ fluorometer and the Qubit ${ }^{\circledR}$ dsDNA HS assay kit (Thermo Fisher Scientific, Inc.). Libraries were multiplexed and clonally amplified by emulsion PCR using the Ion One Touch 2 instrument with the Ion PGM ${ }^{\mathrm{TM}}$ template OT2 200 kit (Thermo Fisher Scientific, Inc.) and sequenced using a $\mathrm{PGM}^{\mathrm{TM}}$ sequencer with the Ion $\mathrm{PGM}^{\mathrm{TM}}$ sequencing 200 kit v2. Quality control was performed using the Ionsphere ${ }^{\mathrm{TM}}$ quality control kit (Thermo Fisher Scientific, Inc.). All steps were performed according to the manufacturer's protocols.

Data analysis. The raw data analysis was performed as previously described $(30,31)$, using Torrent Suite software v4.0.2-v5.10.0 (Thermo Fisher Scientific, Inc.). The coverage analysis was performed using the Coverage Analysis plugin v4.0- v5.10. Cases for which the average base coverage was $<500 x$ were considered non-informative. Detection of mutations was performed using the Variant Caller plugins v4.0-v5.10 (Thermo Fisher Scientific, Inc.). Each mutation was verified in the Integrative Genome Viewer from the Broad Institute (http://www.broadinstitute.org/). Only mutations reported in the COSMIC database (http://cancer.sanger.ac.uk/) were taken into account, while silent or intronic mutations were not reported. Mutations were then classified into three categories based on data obtained from the literature and from the COSMIC database: Mutations with known clinical impact (with a targeted therapy on the market, e.g., BRAF V600 mutation); mutations with potential clinical impact (clinical trials are ongoing, e.g., NRAS, EZH2, BRAF nonV600E); and mutations with unknown clinical impact (e.g., TP53).

The cases with poor-quality sequencing and/or insufficient material for IHC staining were considered non-contributory.

\section{IHC}

IHC procedure. Sections (4 $\mu \mathrm{m}$ thick) of the original FFPE block used for molecular analysis were subjected to anti-BRAF (mutated V600E) IHC staining (cat. no. ab228461; clone VE1; dilution, 1/100; Abcam) and anti-p53 IHC staining (cat. no. M7001; clone DO-7; dilution, 1/200; Agilent Technologies, Inc.) on a Dako Omnis (Agilent Technologies, 

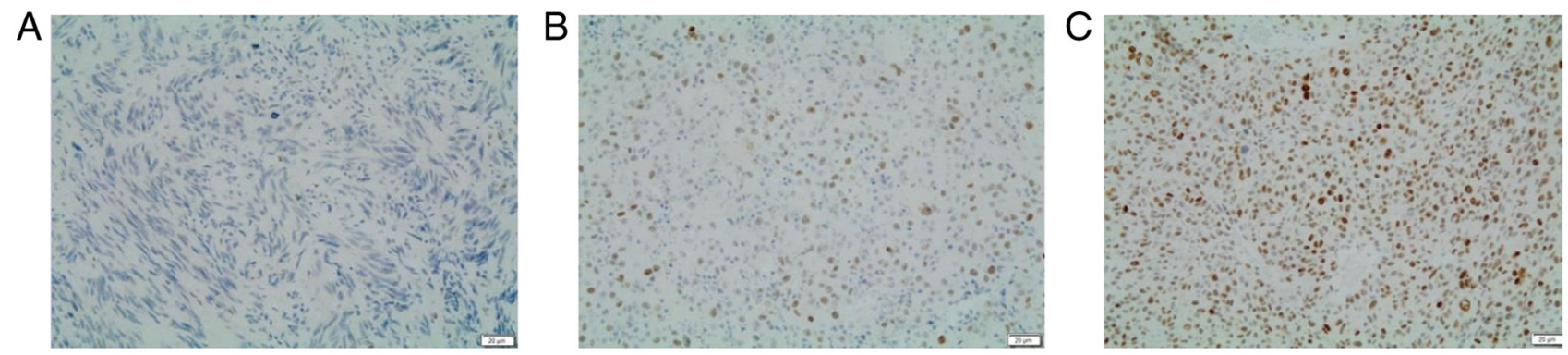

Figure 1. Representative images for three-tier score for immunohistochemical assessment of p53. (A) Score 0-no staining (loss of expression); (B) score $1-\leq 25 \%$ of cells with variable, heterogeneous cytoplasmic and nuclear staining (wild-type); (C) score 2->25\% of cells with high homogeneous nuclear staining (overexpression). Hematoxylin counterstaining was applied and positive staining is brown (original magnification, x200; scale bar, $20 \mu \mathrm{m}$ ).

A

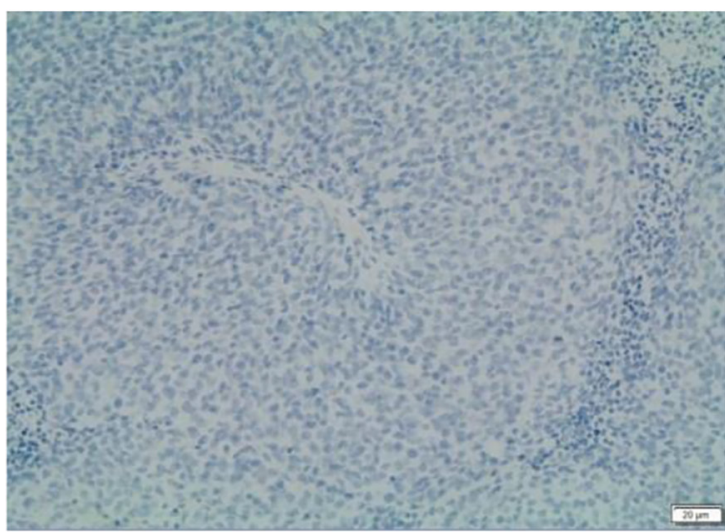

$\mathrm{B}$

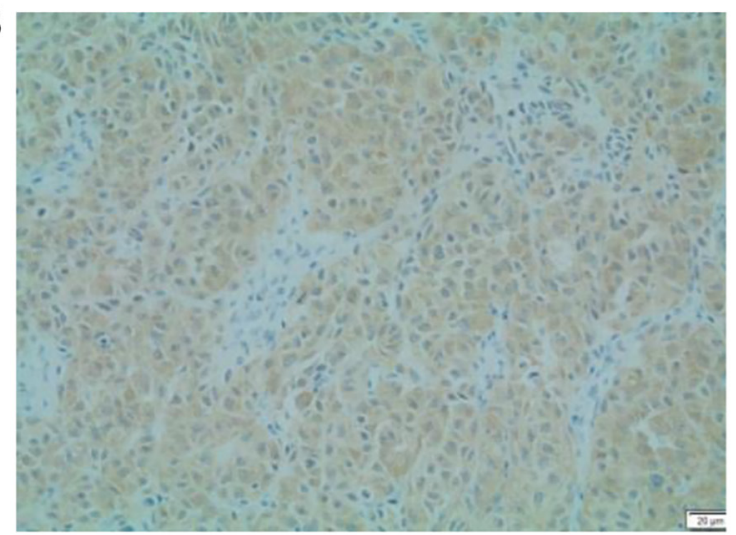

Figure 2. Qualitative assessment score for BRAF V600E immunohistochemistry. (A) Negative-no cytoplasmic staining; (B) positive-presence of cytoplasmic staining. Hematoxylin counterstaining was applied and positive staining is brown (original magnification, x200; scale bar, $20 \mu \mathrm{m}$ ).

Inc.). Heat-induced epitope retrieval was performed using Dako Target Retrieval Solution pH 9 (cat. no. GV804; Agilent Technologies, Inc.) $30 \mathrm{~min}$ at $97^{\circ} \mathrm{C}$, followed by primary antibody incubation $20 \mathrm{~min}$ at $32^{\circ} \mathrm{C}$ and detection with Dako Envision Flex detection system (cat. no. GV800; Agilent Technologies, Inc.) according to the manufacturer's protocol. The sections were counterstained with hematoxylin (cat. no. GC808; Agilent Technologies, Inc.). Control tissues (tonsil for p53 IHC and BRAF V600E mutated tumor for BRAF IHC) and universal negative control antibody (negative control Mouse IgG1; cat. no. X0931; dilution, 1/200; Agilent Technologies, Inc.) were processed in parallel with tissues exposed to the primary as described above.

Pathology scoring. Immunostained slides were evaluated by two trainee pathologists (one and two years of experience) blinded to the molecular data. For discordant cases, a third pathologist ( $>10$ years of experience) blinded to previous results re-evaluated the $\mathrm{p} 53$ and/or BRAF V600E IHC staining.

A semi-quantitative assessment of p53 IHC (Fig. 1) expression was performed by using a three-tier score as previously described (32): 0-no staining (loss of expression); $1-\leq 25 \%$ of cells with variable, heterogeneous cytoplasmic and nuclear staining (wild-type); $2->25 \%$ of cells with high homogeneous nuclear staining (overexpression). This semi-quantitative evaluation is similar to the ones used by Kastelein et al (17) in Barrett's esophagus or Guedes et al (20) in prostate cancer.
A qualitative assessment of BRAF VE1 (Fig. 2) was used as previously described (10-13): Negative-no cytoplasmic staining; positive-presence of cytoplasmic staining.

Pathological data. The pathological data collected were sample type (biopsy/cell block/surgical resection), tumor sample site (skin/conjunctiva/brain/subcutaneous tissue/ liver/lymph node/adrenal glands/digestive tract/lung/ undetermined), IHC analysis (sufficient/insufficient material), IHC BRAF V600E (positive/negative), IHC p53 (no staining/ wild-type/overexpression/non-contributory), NGS analysis (contributory/non-contributory), results of NGS (gene mutation, exon, coverage, \% of mutated DNA, clinical impact). Non-contributory IHC means lack of positive internal control, while non-contributory NGS means that the sequencing quality was too poor to allow for analysis. Clinicopathological data, age and sex distributions were not available for the present study in line with the Ethics Committee consent.

Cost analysis. Information regarding the costs associated with IHC using BRAF VE1 AB Idylla ${ }^{\mathrm{TM}}$ (PCR-based method) and NGS were retrieved. A comparison of the costs was performed between these three methods.

Statistical analysis. The results from IHC and NGS testing were cross-tabulated and, using the online calculator provided by Medcalc (https://www.medcalc.org/calc/diagnostic_test. php), the sensitivity, specificity, accuracy, positive predictive 
Table I. Results of immunohistochemical assessment of p53 and BRAF V600E by pathologists.

\begin{tabular}{lccc}
\hline Protein/result & 1st Pathologist & 2nd Pathologist & Final result \\
\hline p53 & & & $1(3)$ \\
0 (loss of expression) & $0(0)$ & $25(73.5)$ & $22(64.6)$ \\
1 (wild-type) & $23(67.6)$ & $7(20.5)$ & $10(29.4)$ \\
2 (overexpression) & $10(29.4)$ & $1(3)$ & $1(3)$ \\
Non-contributory & $1(3)$ & $30(88.2)$ & $30(88.2)$ \\
BRAF V600E & $30(88.2)$ & $4^{\mathrm{b}}(11.8)$ & $4(11.8)$ \\
Absence of staining & $4^{\mathrm{b}}(11.8)$ & & \\
Presence of staining & & & \\
\hline
\end{tabular}

${ }^{\mathrm{a}}$ Non-contributory p53 IHC means absence of internal positive cells; ${ }^{\mathrm{b}}$ Two of the cases were discordant and reviewed by the third pathologist. Values are expressed as n (\%).

value and negative predictive value were calculated. The association between NGS and IHC was performed using Fisher's exact test with Statistica ${ }^{\circledR}$ (StatSoft). $\mathrm{P}<0.05$ was considered to indicate statistical significance.

To assess the inter-observer coefficient, Cohen's unweighted $\kappa$ coefficient was calculated by running an online test, provided by Vassarstats (http://vassarstats.net/kappa.html). The scale used for the interpretation of the $\kappa$ coefficient was the following: 0-0.2, slight agreement; 0.21-0.40, fair agreement; 0.41-0.60, moderate agreement; 0.61-0.80, substantial agreement; and 0.81-1, almost perfect agreement.

\section{Results}

IHC results and inter-observer concordance. The IHC staining was performed on 34 of 37 cases (three cases did not have sufficient material for immunostaining). A total of 34 cases were further considered for statistical analyses. The IHC profile assessment for p53 and BRAF V600E is summarized in Table I.

Regarding p53, six of the 34 cases were discordant (17.6\%) and were reviewed by a third pathologist, who agreed three times with the first pathologist and three times with the second. One case was not assessable due to the lack of internal positive control. The $\kappa$ coefficient for evaluating the inter-observer concordance was 0.554 , suggesting a moderate agreement.

Concerning BRAF V600E, two of 34 analyzed cases were discordant $(5.90 \%)$ and were reviewed by a third pathologist, who agreed with the first pathologist in both cases. The Cohen unweighted $\kappa$ coefficient was 0.717 , which represents a substantial agreement.

NGS results and NGS-IHC correlation. Sequencing was optimal in 36 out of 37 cases (97.3\%). It failed in one sample $(2.7 \%)$ due to poor quality of sequencing (non-contributory). No mutation in the gene panel was detected for 5 of the 36 cases (13.9\%), 15 (41.7\%) harbored a BRAF mutation and $16(44.4 \%)$ had other mutations than BRAF (potentially clinically actionable mutations or mutations with unknown impact). Of the 15 cases harboring a BRAF mutation, eight were V600E (two cases had insufficient residual tissue for IHC staining, leading to six cases included for NGS-IHC correlation analysis),
Table II. Cross-tabulated results for tumor suppressor protein 53 gene and BRAF V600E mutation status determined by IHC and NGS.

\begin{tabular}{lrc}
\hline & \multicolumn{2}{c}{ NGS result } \\
\cline { 2 - 3 } IHC result & Non-mutated & Mutated \\
\hline p53 & 19 & 2 \\
Wild-type & 8 & 3 \\
Aberrant expression & & \\
BRAF V600E & 27 & 2 \\
No staining & 0 & 4 \\
Presence of staining & & \\
\hline
\end{tabular}

Loss of expression and overexpression of the $\mathrm{p} 53$ protein were considered as positive results (aberrant IHC result); physiological protein expression according to IHC indicated wild-type p53 expression. IHC, immunohistochemistry; NGS, next-generation sequencing.

followed by three V600 non-E (two V600K and one V600R), two D594N, one K601E and one K601N. The most frequent mutations among the non-BRAF mutations were NRAS $(19.4 \%$, 7/36) and TP53 (13.9\%, 5/36). BRAF V600E and TP53 mutations were not mutually exclusive and one case harbored $B R A F$ V600E and TP53 mutations.

When performing the statistical analysis, loss of expression and overexpression of the p53 protein on IHC analysis was considered as a positive result (aberrant IHC result-expression of mutation in the tumor suppressor gene TP53) and the non-contributory cases were eliminated from the analysis. NGS was the reference technique for the analysis of both ABs. NGS and IHC results were available in 32 cases for the evaluation of TP53 protein expression and in 33 cases regarding $B R A F$ V600E protein expression. The cross-tabulated results of the NGS and IHC assessment are summarized in Table II.

Considering the NGS technique to be the reference for the TP53 mutation status, IHC had an overall diagnostic accuracy of $68.8 \%$ for the TP53 mutation with $31.2 \%$ of cases being misclassified (10/32). The overall sensitivity of IHC was $60 \%$ and two cases were false-negative. The overall specificity of 
Table III. NGS-IHC correlation results for both genes tested.

\begin{tabular}{|c|c|c|c|c|}
\hline \multirow[b]{2}{*}{ NGS-IHC correlation } & \multicolumn{2}{|c|}{ TP53 } & \multicolumn{2}{|c|}{$B R A F$ V6OOE } \\
\hline & Value $(\%)$ & $95 \% \mathrm{CI}$ & Value $(\%)$ & $95 \% \mathrm{CI}$ \\
\hline Diagnostic accuracy & 68.8 & $50.0-83.9$ & 93.9 & $79.8-99.3$ \\
\hline Overall sensitivity & 60.0 & $14.7-94.7$ & 66.7 & $22.3-95.7$ \\
\hline Overall specificity & 70.4 & $49.8-86.3$ & 100.0 & $87.2-100.0$ \\
\hline Positive predictive value & 27.3 & $13.0-48.5$ & 100.0 & $51.0-100.0$ \\
\hline Negative predictive value & 90.5 & $76.0-96.6$ & 93.1 & $81.3-97.7$ \\
\hline P-value & \multicolumn{2}{|c|}{0.3098} & \multicolumn{2}{|c|}{0.0004} \\
\hline
\end{tabular}

The NGS technique served as the reference technique (gold standard). The table summarizes all of the values obtained for diagnostic accuracy, overall sensitivity, overall specificity and positive and negative predictive value for each antibody. TP53, tumor (suppressor) protein 53 gene; IHC, immunohistochemistry; NGS, next-generation sequencing.

IHC was $70.4 \%$ and eight cases were false-positive. The positive predictive value of IHC for p53 was $27.3 \%$ (3/11), while the negative predictive value was $90.5 \%$ (19/21). No statistically significant association between the two diagnostic methods was obtained $(\mathrm{P}=0.3098)$.

Considering the NGS technique to be the reference for the $B R A F$ V600E mutation status, IHC had an overall diagnostic accuracy of $93.9 \%$ for the BRAF V600E mutation with $6.1 \%$ of cases being misclassified (2/33). These two misclassified cases were cases harboring a BRAF V600E mutation and negative BRAF VE1 IHC staining. The overall sensitivity of IHC was $66.7 \%$ and two cases were false-negative. The overall specificity for IHC was $100 \%$ with no false-positive results obtained. The positive predictive value of BRAF VE1 was $100 \%$, while the negative predictive value was $93.1 \%(27 / 29)$. A statistically significant association between the two diagnostic methods was determined $(\mathrm{P}=0.0004)$. All related results are summarized in Table III.

Cost-effectiveness analysis for BRAF status testing. In our laboratory, the $B R A F V 600 E$ status may be tested by IHC, Idylla $^{\mathrm{TM}}$ (PCR-based method) or NGS. It was decided to perform the cost analysis only based on the reagents' cost to ensure harmonized results. Amortization costs, maintenance or human resources were not considered, as these elements vary among laboratories. It should be noted that the fact that reagents' costs may also vary depending on the test volume, supplier or country, so that the present cost-effectiveness analysis is only indicative. All of the costs per test for each technique are summarized in Tables SII and SIII. The cost per patient of an NGS analysis considering only the reagents is $\sim 280 €$, that of Idylla ${ }^{\mathrm{TM}} 120 €$ and BRAF VE1 IHC costs $\sim 22 €$. For the present case series, several simulations were performed and it was concluded that performing BRAF V600E IHC staining for all cases first followed by NGS for negative BRAF V600E IHC cases saves $\sim 5 \%$ of costs compared to performing only NGS. Performing Idylla ${ }^{\mathrm{TM}}$ DNA-testing only was revealed to cost less than performing BRAF V600E IHC staining for all cases first followed by Idylla ${ }^{\mathrm{TM}}$. However, in larger case series, IHC staining followed by a DNA-based test for negative cases would cost less than only DNA-based testing.

\section{Discussion}

Melanoma is the most aggressive form of skin cancer with 200,000 new cases diagnosed each year worldwide (33). The identification of $B R A F$ mutations through DNA sequencing in the early 2000s led to a revolution in the treatment of melanoma, allowing the development of therapies targeting the BRAF oncogene and applications of other kinase inhibitors (34). The $B R A F$ mutation status is critical for advanced-stage melanoma and molecular testing should be performed routinely for stage III and IV (unresectable and metastatic) (35) to promptly start the therapy, particularly in melanomas with aggressive behavior. Several $B R A F$ mutation tests are available: DNA-based tests (including PCR and NGS) and one AB-based test for mutant BRAF V600E protein using the mouse monoclonal VE1 clone to detect the protein expression by IHC.

$B R A F$ mutations are observed to occur in numerous different types of cancer, including thyroid, lung, colon cancers, glioblastomas and certain hematological malignancies (16-22,34). BRAF mutations are identified in 40-60\% of melanomas $(14,34,36)$. $B R A F$ V600E is the most common mutation and it accounts for $60-90 \%$ of all BRAF mutations, followed by $V 600 \mathrm{~K}$ mutation and $V 600 D / R$ accounting for $10-30$ and $3 \%$, respectively $(37,38)$. The present results overlap with those described in the literature, as $B R A F$ mutations were identified in $\sim 40 \%$ of the present cases and among the mutated cases, V600E mutation accounts for $53.3 \%$ of cases, followed by $V 600 K$ mutation with $13.3 \%$. The present study demonstrated a sensitivity of $66.7 \%$ and a specificity of $100 \%$ for the BRAF VE1 IHC as the method of detection for BRAF V600E compared to NGS analysis (considered the gold standard) in FFPE tumor tissue samples. Specimens with the V600 non-E mutation were not immunoreactive with the VE1 clone, as already described $(10,11)$. The present results are comparable to previous validation studies in which a qualitative assessment scale for VE1 staining was employed regarding the specificity (95.4-100\%), but the sensitivity was inferior to previously reported results (94.4-100\%) (10-14).

Different scoring systems have been used for the evaluation of $B R A F$ by IHC (two or more than two categories, based on the intensity of the IHC staining or based on the pattern of staining) in previous studies, leading to major 
difficulties in comparing the results. For instance, Lo et al (10), Long et al (11), Colomba et al (12) and Boursault et al (13) used the same evaluation method as that employed in the present study, while Marin et al (39) and Pearlstein et al (40) scored BRAF IHC expression in more than two categories. The establishment of an objective and widely accepted consensus scoring system will lead to the uniformization of results and a decrease in interpretation discrepancies and bias.

The inferior sensitivity may be explained by the small number of cases harboring BRAF V600E included in the present study. Immunostaining with BRAF VE1 AB did not produce any false-positive final result. At the first assessment, one pathologist misclassified a negative case as positive on IHC. This false-positive result may be explained by the lack of experience of the trainee pathologist and may be remedied by providing training programs to correctly evaluate IHC profiles. In the present study, two false-negative results occurred, characterized by the absence of IHC staining but $B R A F$ V600E mutation on NGS. In one of the cases, the interpretation of immunostaining was difficult due to the presence of a high quantity of melanin. In order to increase the specificity of the test, the brown chromogen may be replaced with red chromogen (40). Discordance between pathologists in assessing the BRAF VE1 IHC was determined in two of the 34 cases where a third pathologist's opinion was required. The Cohen's $\kappa$ coefficient obtained (0.717) indicates a substantial agreement between the two pathologists. Although molecular testing is considered the gold standard for the detection of $B R A F$ mutations, the monoclonal VE1 $\mathrm{AB}$ is emerging as a reliable option (10-14,40-42). In the present study, a statistically significant association was demonstrated between the IHC and NGS analysis $(\mathrm{P}=0.0004)$, which confirmed the hypothesis that IHC may be used as a surrogate in the evaluation of the BRAF V600E mutation status. The advantages of IHC are as follows: Shorter TAT, lower cost, small quantity of tumor tissue required for detection and the service may be offered by more histopathology departments than the genomics analysis. Relying on the results obtained, the algorithm incorporating both IHC and DNA-based analysis, as previously proposed by Pearlstein et al (40) or the Anglian Cancer Network Protocol (10), may be considered to be used in current clinical practice (Fig. 3). In this algorithm, BRAF VE1 IHC analysis is performed in all cases of unresectable or metastatic melanoma, providing a quick and inexpensive way to detect $B R A F$ V600E mutations. Furthermore, it is the only modality of $B R A F$ mutation detection in cases with limited tumor tissue that may otherwise not be tested by DNA-based analysis due to insufficient material. Afterwards, cases with negative BRAF VE1 staining should be tested by a DNA-based test in order to identify a possible false-negative result or a $B R A F$ mutation other than V600E. The use of the proposed algorithm would offer a cost-saving of $\sim 5 \%$ compared to the NGS test in all cases. However, testing only by Idylla ${ }^{\mathrm{TM}}$ in the present case series compared to prior BRAF VE1 testing followed by Idylla ${ }^{\mathrm{TM}}$ was more economical, but this is highly dependent on the number of cases. Concerning DNA-based testing, the NGS technique provides information beyond $B R A F$, revealing more 'actionable' mutations leading to other therapeutic options. However, NGS is not widely available, its interpretation requires highly trained staff and it has a longer

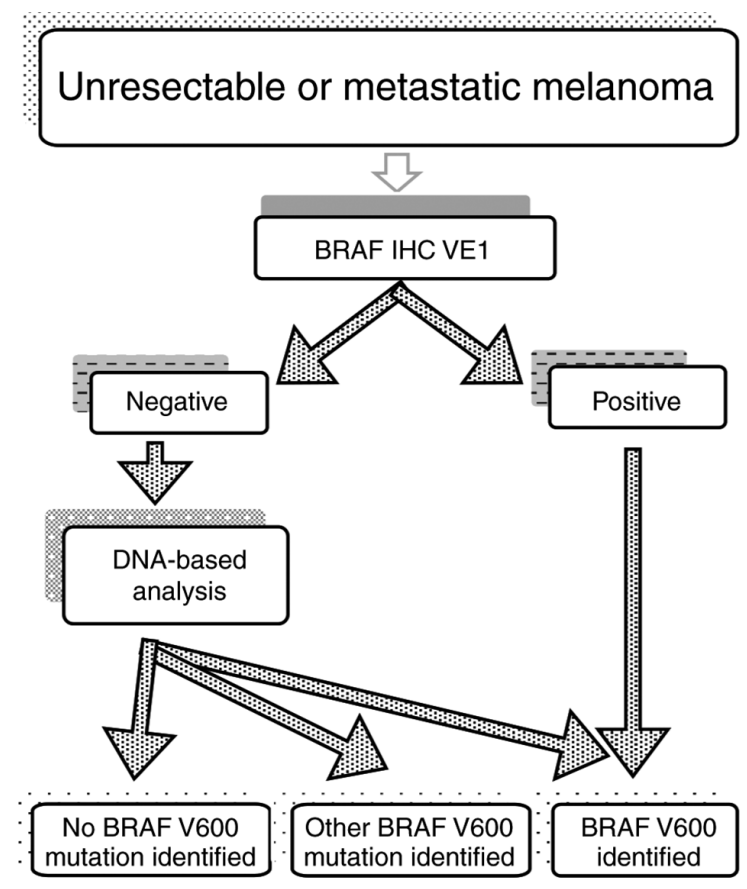

Figure 3. Algorithm proposed for the diagnosis of advanced-stage melanoma. BRAF V600E IHC is routinely applied for all unresectable and metastatic melanomas. In the case of a positive result, targeted therapy may be initiated. DNA-based analysis should be employed when a negative result is obtained in order to further guide the treatment. IHC, immunohistochemistry.

TAT. The Idylla ${ }^{\mathrm{TM}}$ test is a PCR-based test and an alternative to IHC or NGS for BRAF V600 mutation, with the following advantages: It is less laborious, faster and allows the detection of BRAF V600 non-E mutations; however, it has a higher cost and it is not as widely available as IHC (43).

p53 is a transcription factor with a powerful tumor suppressor function. Wild-type p53 is a potent inducer of apoptosis, of cell cycle arrest and of cellular senescence (14). A review published by Lu et al (44) in 2013 highlights the potential role of targeting p53 in melanoma treatment, stating that reactivation of $\mathrm{p} 53$ by simultaneously blocking the p53 E3 ubiquitin ligase, MDM2 and inhibitor of apoptosis-stimulating protein of $\mathrm{p} 53$, together with BRAF V600E inhibition, induces apoptosis and suppresses melanoma growth in cell lines and animal models. Furthermore, a novel hypothesis regarding the importance of the p53 status emerges, stating that TP53 mutation is a potential negative predictor of metastatic melanoma treated by CTLA- 4 blockade $(45,46)$. Thus, it may be worthwhile to routinely evaluate the TP53 status. The frequency of TP53 mutation in melanoma reported in the literature is $\sim 20 \%(9,47)$. A slightly inferior rate $(14 \%)$ was obtained in the present cohort. The association between TP53 mutations and p53 nuclear accumulation remains to be fully elucidated. IHC is used routinely as a surrogate for TP53 mutation analysis in several types of cancer $(17-22,48)$, but its interpretation is at times difficult (48). In the present study, the $\kappa$ coefficient obtained was 0.554 , suggesting a moderate inter-observer concordance, underlining the difficulty in interpreting p53 protein expression. The IHC-NGS association was not statistically significant, indicating that the use of p53 IHC to replace a DNA-based test should not be considered for routine use. In the present study, false-negative (two identified in the present 
cohort) and false-positive (eight identified) results were obtained. The false-negative results harbored different TP53 mutations. Furthermore, not all the mutations are leading to corresponding transcriptional or translational changes in p53 protein products (28). The false-positive results may be explained by the fact that p53 IHC expression may result from malfunction of other components of the p53 pathway other than the gene mutation or the fact that targeted NGS focuses on genomic regions of particular interest, with various regions of the TP53 gene remaining unsequenced. Besides these, the tumors also exhibit genetic heterogeneity (29). Other studies (23-27) also reported no correlation between the protein expression and the TP53 mutational status, suggesting that a direct link between aberrant protein expression of p53 and the presence of TP53 mutation was not able to be established.

As at the present time, determination of the TP53 status is not a standard of care for patients with melanoma in contrast to the $B R A F$ status, expanded molecular testing is not required for all patients. Considering that IHC analysis of p53 is not highly sensitive or specific, when determination of the TP53 mutation will be warranted, it should be assessed by a molecular method.

In conclusion, molecular profiling has become a key piece of information in the management of advanced-stage melanoma with the introduction of 'personalized medicine'. The results obtained demonstrated that IHC staining with BRAF VE1 AB is a reliable surrogate for NGS in identifying the BRAF V600E mutation, becoming an efficient screening tool prior to DNA-based analysis. Loss of p53 expression/overexpression determined by IHC is at times associated with TP53 gene mutations, but it was not possible to establish a root-cause relationship. Aside from that, BRAF VE1 allows the detection of a specific hotspot mutation, while p53 expression on IHC is related to protein expression that may be wild-type but may also mirror a large spectrum of TP53 mutations.

\section{Acknowledgements}

Not applicable.

\section{Funding}

This work was supported by the Fonds Yvonne Boël (Brussels, Belgium).

\section{Availability of data and material}

The original targeted NGS data were submitted to the European Nucleotide Archive. They are available as 'Rusu et al 2021: The use of immunohistochemistry as an accurate tool in the assessment of BRAF V600E and TP53 mutations in primary and metastatic melanoma' with the following study ID: PRJEB42810 (ERP126721) (https://www.ebi. ac.uk/ena/browser/view/PRJEB42810?show=related-records).

\section{Authors' contributions}

$\mathrm{NDH}, \mathrm{IS}, \mathrm{CD}$ and OSC contributed to the conception and design of the study. Material preparation, data collection and IHC analysis were performed by $\mathrm{SRu}, \mathrm{CV}$ and NDH. SDC,
CVC, OB and NDN performed IHC and molecular analyses. ALT, CM and SRo performed the pathological diagnoses. SRU, CV, ND and CD analyzed the data. SRU, CV, NDH and CVC approved the authenticity of the raw data. The first draft of the manuscript was written by SRU and all authors commented on previous versions of the manuscript. All authors read and approved the final manuscript.

\section{Ethics approval and consent to participate}

Approval was granted by the Ethics Committee of the Erasme-Université Libre de Bruxelles (Brussels, Belgium) in May 2019 (no. P2019/310). The Ethics Committee waived the requirement of informed consent under the condition that the researchers check for the absence of any objection of the patient to use their material for research.

\section{Patient consent for publication}

Not applicable.

\section{Competing interests}

The authors declare that they have no competing interests.

\section{References}

1. Glazer AM, Rigel DS, Winkelmann RR and Farberg AS: Clinical diagnosis of skin cancer: Enhancing inspection and early recognition. Dermatol Clin 35: 409-416, 2017.

2. De Vries E, Elder DE, Bray F, Thompson JF, Coebergh JW, Barnhill RL, Cerroni L, van Muijen GNP, Ruiter DJ, Scolyer RA, et al: Chapter 2: Melanocytic tumours. Malignant melanoma: Introduction. In: World Health Organization Classification of Tumours. Pathology and Genetics of Skin Tumours. LeBoit PE, Burg G, Weedon D and Sarasin A (eds). IARC Press, Lyon, pp52-65, 2006.

3. Gershenwald GE, Scolyer RA, Hess KR, Thompson JF, Long GV, Ross MI, Lazar AJ, Atkins MB, Balch CM, Bamhill RL, et al: Part X. Skin-47. Melanoma of the Skin. In: AJCC Cancer Staging Manual, 8th edition. Amin MB, Edge S, Greene F, Byrd DR, Brookland RK, Washington MK, Gershenwald JE, Compton CC, Hess KR, Sullivan DC, et al (eds). Springer, Chicago, IL, pp563-585, 2017.

4. Lazar A and Bastian B: Melanoma. In: McKee's Pathology of the Skin. 4th edition. Calonje JE, Brenn T, Lazar A and McKee P (eds). Elsevier/Saunders, Edinburgh, pp1221-1267, 2012.

5. Matthews N, Li W, Qureshi A, Weinstock M and Cho E: Epidemiology of melanoma. In: Cutaneous Melanoma: Etiology and Therapy. Ward WH and Farma JM (eds) Codon Publications, Brisbane, pp3-23, 2017.

6. World Cancer Research Fund, American Institute for Cancer Research: Worldwide Cancer Data, Global Cancer Statistics for the most common cancers. WCRF International, London, 2019. https://www.wcrf.org/dietandcancer/cancer-trends/worldwide-cancer-data. Accessed May 22, 2019.

7. Curtin JA, Fridlyand J, Kageshita T, Patel HN, Busam KJ, Kutzner H, Cho KW, Aiba S, Brocker EB, LeBoit PE, et al: Distinct sets of genetic alterations in melanoma. N Engl J Med 353: 2135-2147, 2005.

8. Palmieri G, Ombra M, Colombino M, Casula M, Sini M, Manca A, Paliogiannis P, Ascierto PA and Cossu A: Multiple molecular pathways in melanomagenesis: Characterization of therapeutic targets. Front Oncol 5: 183, 2015.

9. Giehl K: Oncogenic Ras in tumor progression and metastasis. Biol Chem 386: 193-205, 2005.

10. Lo MC, Paterson A, Maraka J, Clark R, Goodwill J, Nobes J, Garioch J, Moncrieff M, Rytina E and Igali L: A UK feasibility and validation study of the VE1 monoclonal antibody immunohistochemistry stain for BRAF-V600E mutations in metastatic melanoma. Br J Cancer 115: 223-227, 2016. 
11. Long GV, Wilmott JS, Capper D, Preusser M, Zhang YX, Thompson JF, Kefford RF, von Deimling A and Scolyer RA: Immunohistochemistry is highly sensitive and specific for the detection of V600E BRAF mutation in melanoma. Am J Surg Pathol 37: 61-65, 2013.

12. Colomba E, Helias-Rodzewicz Z, Von Deimling A, Marin C, Terrones N, Pechaud D, Surel S, Côté JF, Peschaud F, Capper D, et al: Detection of BRAF p.V600E mutations in melanomas: Comparison of four methods argues for sequential use of immunohistochemistry and pyrosequencing. J Mol Diagn 15: 94-100, 2013.

13. Boursault L, Haddad V, Vergier B, Cappellen D, Bellocq JP, Jouary T and Merlio JP: Homogénéité et conservation du statut BRAF entre mélanome primitif et métastases déterminées par immunohistochimie et biologie moléculaire. Ann Dermatol Venereol 140: S396, 2013.

14. Tetzlaff MT, Pattanaprichakul P, Wargo J, Fox PS, Patel KP, Estrella JS, Broaddus RR, Williams MD, Davies MA, Routbort MJ, et al: Utility of BRAF V600E immunohistochemistry expression pattern as a surrogate of BRAF mutation status in 154 patients with advanced melanoma. Hum Pathol 46: 1101-1110, 2015.

15. Box NF, Vukmer TO and Terzian T: Targeting p53 in melanoma. Pigment Cell Melanoma Res 27: 8-10, 2014.

16. Köbel M, Piskorz AM, Lee S, Lui S, LePage C, Marass F, Rosenfeld N, Mes Masson AM and Brenton JD: Optimized p53 immunohistochemistry is an accurate predictor of TP53 mutation in ovarian carcinoma. J Pathol Clin Res 2: 247-258, 2016.

17. Kastelein F, Biermann K, Steyerberg EW, Verheij J, Kalisvaart M, Looijenga LH, Stoop HA, Walter L, Kuipers EJ, Spaander MC, et al: Aberrant p53 protein expression is associated with an increased risk of neoplastic progression in patients with Barrett's oesophagus. Gut 62: 1676-1683, 2013.

18. Rodrigues NR, Rowan A, Smith ME, Kerr IB, Bodmer WF, Gannon JV and Lane DP: p53 mutations in colorectal cancer. Proc Natl Acad Sci USA 87: 7555-7559, 1990.

19. Iggo R, Gatter K, Bartek J, Lane D and Harris AL: Increased expression of mutant forms of $\mathrm{p} 53$ oncogene in primary lung cancer. Lancet 335: 675-679, 1990

20. Guedes LB, Almutairi F, Haffner MC, Rajoria G, Liu Z, Klimel S, Zoino R, Yousefi K, Sharma R, De Marzo AM, et al: Analytic, preanalytic, and clinical validation of p53 IHC for detection of TP53 missense mutation in prostate cancer. Clin Cancer Res 23: 4693-4703, 2017

21. Lepelley P,Preudhomme C, Vanrumbeke M, Quesnel B, Cosson A and Fenaux P: Detection of p53 mutations in hematological malignancies: Comparison between immunocytochemistry and DNA analysis. Leukemia 8: 1342-1349, 1994.

22. Dobes P, Podhorec J, Coufal O, Jureckova A, Petrakova K, Vojtesek B and Hrstka R: Influence of mutation type on prognostic and predictive values of TP53 status in primary breast cancer patients. Oncol Rep 32: 1695-1702, 2014.

23. Albino AP, Vidal MJ, McNutt NS, Shea CR, Prieto VG, Nanus DM, Palmer JM and Hayward NK: Mutation and expression of the p53 gene in human malignant melanoma. Melanoma Res 4: 35-45, 1994.

24. Akslen LA, Monstad SE, Larsen B, Straume O and Ogreid D: Frequent mutations of the p53 gene in cutaneous melanoma of the nodular type. Int J Cancer 79: 91-95, 1998.

25. Sparrow LE, English DR, Heenan PJ, Dawkins HJ and Taran J: Prognostic significance of p53 over-expression in thin melanomas. Melanoma Res 5: 387-392, 1995.

26. Florenes VA, Oyjord T, Holm R, Skrede M, Borresen AL, Nesland JM and Fodstad O: TP53 allele loss, mutations and expression in malignant melanoma. Br J Cancer 69: 253-259, 1994.

27. Weiss J, Heine M, Arden KC, Körner B, Pilch H, Herbst RA and Jung EG: Mutation and expression of TP53 in malignant melanomas. Recent Results Cancer Res 139: 137-154, 1995.

28. Soussi T, Legros Y, Lubin R, Ory K and Schlichtholz B: Multifactorial analysis of p53 alterations in human cancer: A review. Int J Cancer 57: 1-9, 1994.

29. Hussein MR: The TP53 tumor supressor gene and melanoma tumorigenesis: Is there a relationship? Tumor Biol 25: 200-207, 2004.

30. Le Mercier M, D'Haene N, De Nève N, Blanchard O, Degand C, Rorive $\mathrm{S}$ and Salmon I: Next-generation sequencing improves the diagnosis of thyroid FNA specimens with indeterminate cytology. Histopathology 66: 215-224, 2015.
31. D'Haene N, Le Mercier M, De Nève $N$, Blanchard $O$, Delaunoy M, El Housni H, Dessars B, Heimann P, Remmelink M, Demetter P, et al: Clinical validation of targeted next generation sequencing for colon and lung cancers. PLoS One 10: e0138245, 2015.

32. Lebrun L, Milowich D, Le Mercier M, Allard J, Van Eycke YR, Roumeguere T, Decaestecker C, Salmon I and Rorive S: UCA1 overexpression is associated with less aggressive subtypes of bladder cancer. Oncol Rep 40: 2497-2506, 2018.

33. Miraflor AP, de Abreu FB, Peterson JD, Turner SA, Amos CI, Tsongalis GJ and Yan S: Somatic mutation analysis in melanoma using targeted next generation sequencing. Exp Mol Pathol 103: 172-177, 2017.

34. Holderfield M, Deuker MM, McCormick F and McMahon M: Targeting RAF kinases for cancer therapy: BRAF-mutated melanoma and beyond. Nat Rev Cancer 4: 455-467, 2014.

35. Coit DG, Thompson JA, Albertini MR, Barker C, Carson WE, Contreras C, Daniels GA, DiMaio D, Fields RC, Fleming MD, et al: Cutaneous Melanoma, Version 2.2019, NCCN clinical practice guidelines in oncology. J Natl Compr Canc Netw 17: 367-402, 2019.

36. Lee JH, Choi JW and Kim YS: Frequencies of BRAF and NRAS mutations are different in histological types and sites of origin of cutaneous melanoma: A meta-analysis. Br J Dermatol 164: 776-784, 2011

37. Kudchadkar R, Paraiso KH and Smalley KS: Targeting mutant BRAF in melanoma: Current status and future development of combination therapy strategies. Cancer J 18: 124-131, 2012.

38. Thiel A, Moza M, Kytola S, Orpana A, Jahkola T, Hernberg M, Virolainen S and Ristimaki A: Prospective immunohistochemical analysis of BRAF V600E mutation in melanoma. Hum Pathol 46: 169-175, 2015.

39. Marin C, Beauchet A, Capper D, Zimmermann U, Julie C, Ilie M, Saiag P, von Deimling A, Hofman P and Emile JF: Detection of BRAF p.V600E mutations in melanoma by immunohistochemistry has a good interobserver reproducibility. Arch Pathol Lab Med 138: 71-75, 2014.

40. Pearlstein MV, Zedek DC, Ollila DW, Treece A, Gulley ML, Groben PA and Thomas NE: Validation of the VE1 Immunostain for the BRAF V600E mutation in melanoma. J Cutan Pathol 41: 724-732, 2014.

41. Schirosi L, Strippoli S, Gaudio F, Graziano G, Popescu O, Guida M, Simone G and Mangia A: Is immunohistochemistry of BRAF V600E useful as a screening tool and during progression disease of melanoma patients? BMC Cancer 16: 905, 2016.

42. Cheng L, Lopez-Beltran A, Massari F, MacLennan GT and Montironi R: Molecular testing for BRAF mutations to inform melanoma treatment decisions: A move toward precision medicine. Mod Pathol 31: 24-38, 2018

43. Bisschop C, Ter Elst A, Bosman LJ, Platteel I, Jalving M, van den Berg A, Diepstra A, van Hemel B, Diercks GFH, Hospers GAP and Schuuring E: Rapid BRAF mutation tests in patients with advanced melanoma: Comparison of immunohistochemistry, Droplet Digital PCR, and the Idylla Mutation Platform. Melanoma Res 28: 96-104, 2018.

44. $\mathrm{Lu} \mathrm{M}$, Miller P and $\mathrm{Lu} \mathrm{X}$ : Restoring the tumour suppressive function of $\mathrm{p} 53$ as a parallel strategy in melanoma therapy. FEBS Lett 588: 2616-2621, 2014

45. Onyshchenko M: The puzzle of predicting response to immune checkpoint blockade. EBioMedicine 33: 18-19, 2018.

46. Xiao W, Du N, Huang T, Guo J, Mo X, Yuan T, Chen Y, Ye T, $\mathrm{Xu} \mathrm{C}$, Wang W, et al: TP53 mutation as potential negative predictor for response of Anti-CTLA-4 therapy in metastatic melanoma. EBioMedicine 32: 119-124, 2018.

47. Siroy AE, Boland GM, Milton DR, Roszik J, Frankian S, Malke J, Haydu L, Prieto VG, Tetzlaff M, Ivan D et al: Beyond BRAF(V600): Clinical mutation panel testing by next-generation sequencing in advanced melanoma. J Invest Dermatol 135: $508-515,2015$.

48. Murniak B and Hortobagyi T: Immunohistochemical correlates of TP53 somatic mutations in cancer. Oncotarget 7: 64910-64920, 2016.

his work is licensed under a Creative Commons

Attribution-NonCommercial-NoDerivatives 4.0 International (CC BY-NC-ND 4.0) License. 